\title{
COMPOSITION OPERATORS WITH CLOSED RANGE
}

\author{
NINA ZORBOSKA
}

\begin{abstract}
We characterize the closed-range composition operators on weighted Bergman spaces in terms of the ranges of the inducing maps on the unit disc. The method uses Nevanlinna's counting function and Luecking's results on inequalities on Bergman spaces.
\end{abstract}

\section{INTRODUCTION}

A function $\phi$ analytic on the unit disc and mapping the disc into itself induces a composition operator $C_{\phi}$ on the Hardy space $H^{2}$ defined by

$$
C_{\phi} f=f \circ \phi
$$

for all $f$ in $H^{2}$. It follows from Littlewood's inequality that $C_{\phi}$ is bounded on $H^{2}$. The extensive study of composition operators began in the late seventies and a good reference is Cowen's status report (see [3]).

In 1974 Cima, Thompson, and Wogen determined the composition operators on $H^{2}$ that have a closed range using the boundary behaviour of the inducing maps (see [2]). They end their article by posing the problem of characterizing the closed-range composition operators using the properties of the range of the inducing maps on the unit disc rather than the properties on the boundary.

In this paper we give a solution to this problem by using the Nevanlinna counting function and Luecking measure theoretic results on inequalities on Bergman spaces. The result gives a geometrical view of the problem. It determines the closed range composition operator $C_{\phi}$ by means of "thickness" of the subset of the unit disc on which the corresponding determining function $\tau_{\phi}$ (to be defined later) is bounded below.

Main Theorem. An operator $C_{\phi}$ on $H^{2}$ has a closed range if and only if there exists a positive constant $c$ such that the set

$$
G_{c}^{\phi}=\left\{z: \tau_{\phi}(z)>c\right\}
$$

satisfies the following condition:

(*) There exists a constant $\delta>0$ such that

$$
m\left(G_{c} \cap D(\xi, r)\right)>\delta \cdot m(D \cap D(\xi, r))
$$

Received by the editors December 7, 1992 and, in revised form, September 9, 1993.

1991 Mathematics Subject Classification. Primary 47B38, 47A05; Secondary 30H05.

Supported in part by NSERC grant. 
for all $\xi$ in $\partial D$ and $r>0$, where $D(\xi, r)$ is a disc with a center $\xi$ and $a$ radius $r$.

Condition $(*)$ describes the "thickness" in terms of basic sets of type $D(\xi, r)$. We shall point out later that we can choose different types of basic sets (for example, Carleson squares).

In the second section we give the definitions and cite the results that will be used in the proof of the Main Theorem which then comprises the third section. A generalization of the result on weighted Bergman spaces is given in the fourth section. The fifth section is devoted to examples and connections to some other problems.

I would like to thank J. Shapiro for his encouragement and the helpful discussion on the subject that led me to the results in this paper.

\section{Preliminaries}

(a) Nevanlinna counting function. In 1987, Shapiro [8] used for the first time the Nevanlinna counting function of the inducing map to derive conclusions about composition operators. Our solution of the closed range problem relies on the same function.

For an analytic map $\phi$ from the unit disc $D$ into itself, we define the Nevanlinna counting function of $\phi$ by

$$
N_{\phi}(w)=\sum\left\{\log \frac{1}{|z|}: z \in \phi^{-1}(w)\right\}
$$

for all $w$ in $\phi(D) \backslash\{\phi(0)\}$, where $\phi^{-1}(w)$ denotes the set of $\phi$-preimages of $w$ counting the multiplicity, and $N_{\phi}(w)=0$ if $w \notin \phi(D)$.

Let $\tau_{\phi}(w)$ be a function on $D \backslash\{\phi(0)\}$ defined by

$$
\tau_{\phi}(w)=\frac{N_{\phi}(w)}{\log (1 /|w|)} .
$$

From the Littlewood's inequality (see, for example, [8]) it follows that

$$
N_{\phi}(w) \leq \log |(1-\overline{\phi(0)} w) /(\phi(0)-w)|
$$

for all $w$ in $D \backslash\{\phi(0)\}$. If $\phi$ is an inner function, the inequality becomes an equality for all $w$ outside a subset of $D$ with a logarithmic capacity of zero. When $\phi(0)=0$, we get $N_{\phi}(w) \leq \log (1 /|w|)$, i.e., $\tau_{\phi}(w) \leq 1$ for all $w$ in $D \backslash\{0\}$. So, if $\phi(0)=0$ and $\phi$ is inner, we get that $\tau_{\phi}(w)=1$ for all $\omega$ in $D$ outside a subset of $D$ with a logarithmic capacity of zero.

In general, $\tau_{\phi}(w)$ is a nonnegative measurable function that satisfies the submean value property. It is not necessarily a bounded function on $D$, but it is bounded on $D$ minus a neighborhood of $\phi(0)$ and it belongs to $L^{1}\left(\log \left(1 /|z|^{2}\right) d m\right) \quad($ see $[8])$.

(b) Equivalent norm in $H^{2}$. The standard norm in the Hardy space $H^{2}$ is defined by

$$
\|f\|_{2}^{2}=\sup \left\{\frac{1}{2 \pi} \int_{0}^{2 \pi}\left|f\left(r e^{i \theta}\right)\right|^{2} d \theta: 0 \leq r<1\right\},
$$

for $f$ in $H^{2}$. 
Calculations with power series prove that if $m$ is the normalized Lebesgue area measure on $D$, then we can define an equivalent norm on $H^{2}$ by

$$
\|f\|^{2}=\int_{D}\left|f^{\prime}(z)\right|^{2} \log \frac{1}{|z|^{2}} d m(z)+|f(0)|^{2} .
$$

This norm is more suitable when we want to regard $H^{2}$ as being just one of the weighted Bergman spaces. They will be discussed in $\S 4$.

(c) Change of variable formula. As a special case of Stanton's formula for integral means we have the formula

$$
\int\left|f^{\prime} \circ \phi\right|^{2}\left|\phi^{\prime}\right|^{2} \log \frac{1}{|z|^{2}} d m(z)=2 \int\left|f^{\prime}\right|^{2} N_{\phi} d m
$$

for $f$ in $H^{2}$ and $\phi$ (as always) an analytic function on $D$ mapping $D$ into itself (see [8]).

The above equation establishes a connection between the composition operators and the Nevanlinna counting function.

(d) Luecking's inequality. The last essential building block for our result is Luecking's theorem from [4], which we restate for our purposes in the following form.

Theorem (D. Luecking). Let $\tau$ be a bounded nonnegative measurable function in $D$. Then there is a constant $k>0$ such that

$$
\int_{D}\left|f^{\prime}(z)\right|^{2} \tau(z) \log \frac{1}{|z|^{2}} d m(z) \geq k \int_{D}\left|f^{\prime}(z)\right|^{2} \log \frac{1}{|z|^{2}} d m(z)
$$

for all $f$ in $H^{2}$ if and only if there exists a constant $c>0$ such that the set $G_{c}=\{z \in D: \tau(z)>c\}$ satisfies the condition:

$(*)$ There exists a constant $\delta>0$ such that

$$
m\left(G_{c} \cap D(\xi, r)\right)>\delta \cdot m(D \cap D(\xi, r))
$$

for all $\xi$ in $\partial D$ and $r>0$, where $D(\xi, r)$ is a disc with a center $\xi$ and a radius $r$.

In [4] Luecking shows that there are two other ways of restating condition (*) on $G_{c}$ (we shall denote them by $(* *)$ and $\left.(* * *)\right)$ :

(**) There exists $\delta_{1}>0$ and $\eta$ with $0<\eta<1$ such that $m\left(G_{c} \cap D_{\eta}(a)\right)>$ $\delta_{1} \cdot m\left(D_{\eta}(a)\right)$ for all $a$ in $D$, where $D_{\eta}(a)$ is the disc with a center $a$ and a radius $\eta(1-|a|)$.

$(* * *)$ There exists $\delta_{2}>0$ such that $m\left(G_{c} \cap S\right)>\delta_{2} \cdot m(S)$ for all $S=S_{\theta, h}=$ $\left\{r e^{i t}: 1-h<r<1,|\theta-t|<h\right\}$ where $\theta$ is a real number and $0<h<1$.

Note that the sets that satisfy condition $(*)$ are, for example, the unit disc itself (minus any set with area of measure 0 ), any annulus of the form $D \backslash D(0, r)$ with $r<1$ (minus a set with area of measure 0 ) and any set bigger than a set that already satisfies condition $(*)$.

Sets that do not satisfy condition $(*)$ are, for example, the unit disc outside of a neighborhood of a point on $\partial D$ (plus a set with area of measure 0 ), the 
unit disc outside of a disc internally tangent to it (plus a set with area of measure $0)$, and any set smaller than a set that does not satisfy condition $(*)$.

\section{The MaIn Theorem}

We proceed now to present the proof of the Main Theorem by first proving a few lemmas. Since every composition operator is one-to-one, it has a closed range if and only if it is bounded below. We shall prove the "boundedness from below" part by using Luecking's inequality.

Let us first look at the inner functions which play a special role in the closed range problem. By estimations on the norm of $C_{\phi}$, Nordgren proved [7] that if $\phi$ is an inner function then $C_{\phi}$ is bounded from below on $H^{2}$ by $[(1-|\phi(0)|) /(1+|\phi(0)|)]^{1 / 2}$, i.e., $C_{\phi}$ has a closed range.

On the other side, recall that inner functions satisfy the equality in the Littlewood's inequality. That is the main reason why the following lemma holds. The lemma gives another proof of the fact that composition operators induced by inner functions have a closed range on $H^{2}$.

Lemma 3.1. For every inner function $\psi$ there exists a positive constant $c$ such that the set $G_{\psi}^{c}=\left\{z: \tau_{\psi}(z)>c\right\}$ contains an annulus $\{z: R<|z|<1\}$ minus a set of lagorithmic capacity 0 , for some $R$ with $0<R<1$.

Proof. Recall that for $\psi$ inner, we have

$$
N_{\psi}(z)=\log |(1-\overline{\psi(0)} z) /(\psi(0)-z)|
$$

for all $z$ in $D$ except on a set of logarithmic capacity 0 (see [8]). Let $a=\psi(0)$ and

$$
\psi_{a}(z)=(a-z) /(1-\bar{a} z) .
$$

From the identity on disc automorphisms (see Garnett, Bounded functions) it follows that

$$
\frac{1-\left|\psi_{a}(z)\right|^{2}}{1-|z|^{2}}=\frac{1-|a|^{2}}{|1-\bar{a} z|^{2}}
$$

which is greater or equal to $\left(1-|a|^{2}\right) / 4$ since $|1-\bar{a} z|^{2} \leq 4$.

As $|z| \rightarrow 1^{-}, \log \left(1 /|z|^{2}\right)$ behaves similarly as $1-|z|^{2}$, and so, for some $R$, $0<R<1$ and $|z|>R$, we have

$$
\tau_{\psi}(z)=\frac{N_{\psi}(z)}{\log (1 /|z|)}=\frac{\log \left(1 /\left|\psi_{a}(z)\right|\right)}{\log (1 /|z|)} \geq \frac{1-|a|^{2}}{4}
$$

except possibly on a set of logarithmic capacity 0 . Any choice of $c$ between 0 and $\left(1-|a|^{2}\right) / 4$ works.

Note that for $\psi$ inner and $\psi(0)=0$ we get that $\tau_{\psi}(z)=1$ for almost all $z$ in $D \backslash\{0\}$, so for any $c$ with $0<c<1$ we have that $G_{c}^{\psi}=D \backslash\{0\}$ (disregarding sets of logarithmic capacity 0 ).

As before, let $m$ be the normalized Lebesgue area measure on $D$. Define $m_{1}$ to be the measure on $D$ such that

$$
d m_{1}(z)=\log \left(1 /|z|^{2}\right) d m(z) .
$$

Lemma 3.2. Let $\phi$ be analytic in $D$ and let it map $D$ into itself. Then there exists a positive constant $c$ such that the set $G_{c}^{\phi}=\left\{z: \tau_{\phi}(z)>c\right\}$ satisfies 
condition $(*)$ if and only if there exists a positive constant $k$ for which

$$
\int_{D}\left|(f \circ \phi)^{\prime}(z)\right|^{2} d m_{1}(z) \geq k \cdot \int_{D}\left|f^{\prime}(z)\right|^{2} d m_{1}(z)
$$

for all $f$ in $H^{2}$.

Proof. For $f \in H^{2}$, we have that

$$
\begin{aligned}
\int_{D}\left|(f \circ \phi)^{\prime}\right|^{2} d m_{1}(z) & =\int_{D}\left|f^{\prime}(\phi(z))\right|^{2}\left|\phi^{\prime}(z)\right|^{2} d m_{1}(z) \\
& =2 \int_{D}\left|f^{\prime}(z)\right|^{2} N_{\phi}(z) d m(z) \\
& =\int_{D}\left|f^{\prime}(z)\right|^{2} \tau_{\phi}(z) \cdot \log \frac{1}{|z|^{2}} d m(z) \\
& =\int_{D}\left|f^{\prime}(z)\right|^{2} \tau_{\phi}(z) d m_{1}(z) .
\end{aligned}
$$

We may not apply Luecking's inequality directly since the function $\tau_{\phi}(z)$ is not necessarily bounded on $D$. We consider an annulus $\{z: R<|z|<1\}$ on which $\tau_{\phi}(z)$ is bounded, and let

$$
\tau_{\phi}^{R}(z)= \begin{cases}\tau_{\phi}(z), & |z|>R \\ 0, & |z| \leq R\end{cases}
$$

Then we have

$$
\begin{aligned}
\int_{D}\left|f^{\prime}(z)\right|^{2} \tau_{\phi}(z) d m_{1}(z) & \geq \int_{R<|z|<1}\left|f^{\prime}(z)\right|^{2} \tau_{\phi}(z) d m_{1}(z) \\
& =\int_{D}\left|f^{\prime}(z)\right| \tau_{\phi}^{R}(z) d m_{1}(z)
\end{aligned}
$$

where by Luecking's inequality there exists a positive constant $k$ such that

$$
\int_{D}\left|f^{\prime}(z)\right| \tau_{\phi}^{R}(z) d m_{1}(z) \geq k \int_{D}\left|f^{\prime}(z)\right| d m_{1}(z)
$$

if and only if there exists a positive constant $c$ such that the set $G_{c}=\left\{z: \tau_{\phi}^{R}(z)\right.$ $>c\}$ satisfies property $(*)$. Since $G_{c}=G_{c}^{\phi} \cap\{z: R<|z|<1\}$, the last statement is equivalent to the fullfilment of property $(*)$ by the set $G_{c}^{\phi}=\left\{z: \tau_{\phi}(z)>c\right\}$.

Lemma 3.3. If $\phi$ is such that $\phi(0)=0$, then $C_{\phi}$ has a closed range if and only if there exists a positive constant $c$ such that the set $G_{c}^{\phi}=\left\{z: \tau_{\phi}(z)>c\right\}$ satisfies condition $(*)$.

Proof. Denote $H_{0}^{2}=\left\{f: f(0)=0, f \in H^{2}\right\}$. Since $\phi(0)=0, H_{0}^{2}$ is an invariant subspace for $C_{\phi}$. Let $T=C_{\phi} / H_{0}^{2}$. Then, for $f \in H_{0}^{2}$ we have

$$
\|T f\|^{2}=\|f \circ \phi\|^{2}=\int_{D}\left|(f \circ \phi)^{\prime}\right|^{2} d m_{1}+|f(\phi(0))|^{2}=\int_{D}\left|(f \circ \phi)^{\prime}\right|^{2} d m_{1}
$$

and

$$
\|f\|^{2}=\int_{D}\left|f^{\prime}\right|^{2} d m_{1}+|f(0)|^{2}=\int_{D}\left|f^{\prime}\right|^{2} d m_{1}
$$


It follows from Lemma 3.2 that $T$ is bounded below (i.e., that $T$ has a closed range) if and only if $G_{c}^{\phi}$ satisfies $(*)$. But $C_{\phi} 1=1$, and so $C_{\phi}$ has a closed range in $H^{2}$.

We are now ready to deal with the most general case.

Theorem 3.4 (Main Theorem). An operator $C_{\phi}$ on $H^{2}$ has a closed range if and only if there exists a positive constant $c$ such that the set $G_{c}^{\phi}=\left\{z: \tau_{\phi}(z)>c\right\}$ satisfies condition $(*)$.

Proof. The case $\phi(0)=0$ is covered in Lemma 3.3, and so we may consider only $\phi(0) \neq 0$. Let $\psi$ be a disc automorphism with $\psi(\phi(0))=0$ and let $\phi_{1}(z)=(\psi \circ \phi)(z)$. Then $\phi_{1}(0)=0, C_{\phi_{1}}=C_{\phi} \cdot C_{\psi}$, and since the range of $C_{\psi}$ is $H^{2}$, the range of $C_{\phi_{1}}$ is the range of $C_{\phi}$.

Because of Lemma 3.3, to finish the proof we need to show that there is some $c>0$ such that $G_{c}^{\phi}$ satisfies $(*)$ if and only if there is some $c_{1}>0$ such that $G_{c_{1}}^{\phi_{1}}$ satisfies $(*)$, where $G_{c_{1}}^{\phi_{1}}=\left\{z: \tau_{\phi_{1}}(z)>c_{1}\right\}$.

So, let us suppose first that there is $c>0$ such that $G_{c}^{\phi}$ satisfies condition $(*)$. By Lemma 3.2, there exists $k>0$ such that $\int_{D}|(f \circ \phi)|^{\prime} d m_{1} \geq$ $k \int\left|f^{\prime}\right|^{2} d m_{1}$ for all $f \in H^{2}$.

On the other hand, since $\psi$ is an inner function, by Lemma 3.2 and Lemma 3.3 , there exists $k_{1}>0$ such that $\int_{D}\left|(f \circ \psi)^{\prime}\right| d m_{1} \geq k_{1} \int_{D}\left|f^{\prime}\right|^{2} d m_{1}$ for all $f \in H^{2}$.

We get that

$$
\begin{aligned}
\int_{D}\left|\left(f \circ \phi_{1}\right)^{\prime}\right| d m_{1} & =\int_{D}\left|((f \circ \psi) \circ \phi)^{\prime}\right|^{2} d m_{1} \\
& \geq k \int_{D}\left|(f \circ \psi)^{\prime}\right|^{2} d m_{1} \geq k_{1} k \int_{D}\left|f^{\prime}\right|^{2} d m_{1} .
\end{aligned}
$$

But then Lemma 3.2 guarantees the existence of $c_{1}>0$ such that $G_{c_{1}}^{\phi_{1}}$ satisfies condition $(*)$.

The opposite implication follows similarly if we only replace $\psi$ by $\psi^{-1}$ and use the fact that $\phi=\psi^{-1} \circ \phi_{1}$.

\section{Weighted Bergman SPACES}

Let $\alpha>-1$ and define a measure on $D$ by

$$
d m_{\alpha}(z)=\frac{1}{\Gamma(\alpha+1)}\left(\log \frac{1}{|z|^{2}}\right)^{\alpha} d m(z)
$$

where $\Gamma$ is the gamma function. We define a weighted Bergman space $A_{\alpha}^{2}$ to be the set of all functions $f$ analytic on $D$ with

$$
\|f\|_{\alpha}^{2}=\int_{D}|f|^{2} d m_{\alpha}<\infty .
$$

For example $A_{0}^{2}$ is the Bergman space, and the Hardy space $H^{2}$ is the set of functions $f$ analytic in $D$ for which $f^{\prime}$ belongs to $A_{1}^{2}$.

For a function $\phi$ that maps $D$ into itself and for $\alpha \geq 0$ we define a generalized Nevanlinna counting function by

$$
N_{\phi, \alpha}(w)=\sum\left\{(\log (1 /|z|))^{\alpha}: z \in \phi^{-1}(w)\right\}
$$


where $\phi^{-1}(w)$ is as before and $N_{\phi, \alpha}(w)=0$ for $w \notin \phi(D)$.

Define also

$$
\tau_{\phi, \alpha}(w)=\frac{N_{\phi, \alpha}(w)}{(\log (1 /|w|))^{\alpha}}
$$

for $w \in D \backslash\{\phi(0)\}$.

For $\alpha \geq 1$ the similar (extended) Littlewood inequality is true:

$$
N_{\phi, \alpha}(w) \leq\left(\log \left|\frac{1-\overline{\phi(0)} w}{\phi(0)-w}\right|\right)^{\alpha}
$$

and we have the modified change of variable formula for $\alpha>1$ :

$$
\int\left|(f \circ \phi)^{\prime}\right|\left|\phi^{\prime}\right|^{2} d m_{\alpha}=c_{\alpha} \int\left|f^{\prime}\right|^{2} N_{\phi, \alpha} d m,
$$

for $f$ in $A_{\alpha-2}^{2}$ and $c_{\alpha}$ a constant (for details see [8]).

In this generalized context Lemma 3.1 is not true any more. The reason for that is that the extended Littlewood's inequality is not necessarily an equality for inner functions.

Note that there exists a compact composition operator on a weighted Bergman space induced by an inner function (see [5]), i.e., inner functions can induce composition operators on weighted Bergman spaces that do not have a closed range.

We can overcome this obstacle because, first of all, for the proof of the Main Theorem we needed Lemma 3.1 only for the case when $\phi$ is a discautomorphism, and secondly, Lemma 3.1 is true if we impose univalence as an extra condition. In the univalent case

$$
N_{\phi, \alpha}(w)=\left(N_{\phi}(w)\right)^{\alpha} \quad \text { and } \quad \tau_{\phi, \alpha}(w)=\left(\tau_{\phi}(w)\right)^{\alpha}
$$

so that Lemma 3.1 works with the constant $c$ being replaced by the constant $c^{\alpha}$.

Since Luecking's inequality can also be generalized to weighted Bergman spaces (see [4]), the generalization of our Main Theorem follows.

Note that for $\phi$ analytic on $D$ and mapping $D$ into itself, the composition operator $C_{\phi}$ is bounded on all weighted Bergman spaces.

Theorem 4.1. The composition operator $C_{\phi}$ on a weighted Bergman space $A_{\alpha}^{2}$ $(\alpha>-1)$ has a closed range if and only if there exists a positive constant $c$ such that the set $G_{c}^{\phi, \alpha}=\left\{z: \tau_{\phi, \alpha+2}(z)>c\right\}$ satisfies property $(*)$.

Observe that the case of the space $H^{2}$ is not covered by Theorem 4.1 but is rather its limiting case where $\alpha$ approaches -1 .

In the following corollaries we give the relation between the closed-range problems in $H^{2}$ and in $A_{\alpha}^{2}$.

Corollary 4.2. If $C_{\phi}$ has a closed range in $A_{\alpha}^{2}$ for some $\alpha>-1$, then $C_{\phi}$ has a closed range in $\mathrm{H}^{2}$.

Proof. Since $\tau_{\phi, \alpha+2}(w) \leq\left(\tau_{\phi}(w)\right)^{\alpha+2}$, we have that, for $c>0$, the set $G_{c}^{\phi, \alpha+2}$ is included in the set $G_{c_{1}}^{\phi}$, where $c_{1}=\sqrt[\alpha+2]{c}$. So, if $G_{c}^{\phi, \alpha+2}$ satisfies property $(*)$, then so does the set $G_{c_{1}}^{\phi}$. 
Note that the converse statement is not true: MacCluer and Shapiro [5] gave an example of an inner function for which $C_{\phi}$ is compact on all $A_{\alpha}^{2}, \alpha>-1$. So $C_{\phi}$ does not have a closed range on $A_{\alpha}^{2}, \alpha>-1$, but $C_{\phi}$ has a closed range on $H^{2}$.

Corollary 4.3. Let $\phi$ be univalent. Then the following statements are equivalent:

(i) $C_{\phi}$ has a closed range on $H^{2}$.

(ii) $C_{\phi}$ has a closed range on $A_{\alpha}^{2}$ for some $\alpha, \alpha>-1$.

(iii) $C_{\phi}$ has a closed range on $A_{\alpha}^{2}$ for all $\alpha, \alpha>-1$.

Proof. The proof follows from the fact that if $\phi$ is univalent, then we have that $N_{\phi, \gamma}(w)=\left(N_{\phi}(w)\right)^{\gamma}$ and so $\tau_{\phi, \gamma}(w)=\left(\tau_{\phi}(w)\right)^{\gamma}$, where $\gamma$ is any real number.

\section{EXAMPLES AND REMARKS}

Cima, Thompson, and Wogen [2] have several examples of functions which are analysed with regard to the closed-range problem of the induced composition operators on $H^{2}$. To apply their Main Theorem they needed the estimation on Radon-Nikodym derivative of the appropriate measures and that is possible, for example, in the case when $\phi$ is a conformal map. Then the limit of its derivative, as the argument tends to the boundary of $D$, can be described.

With our result we can easily dispose of at least two general types of examples with no restriction on multiplicity. These include some of the examples in [2].

Example 1. If the range of $\phi$ on $D$ misses a neighborhood of a point on the unit circle then $C_{\phi}$ does not have a closed range on $H^{2}$.

The conclusion follows trivially from the Main Theorem and from the observation that if the disc $D(\xi, r)$ is chosen to be included in the missed neighborhood of the point $\xi$ on $\partial D$, then $\tau_{\phi}(z)=0$ on $D(\xi, r)$ and so $m\left(G_{c}^{\phi} \cap\right.$ $D(\xi, r))=0$ for every positive constant $c$.

Example 2. If the range of $\phi$ on $D$ has a hole that includes a disc internally tangent to the unit disc, then $C_{\phi}$ does not have a closed range on $H^{2}$.

In this case it is more appropriate to replace property $(*)$ in the Main Theorem by the equivalent property $(* *)$. Recall that $D_{\eta}(a)$ is the disc with radius $\eta(1-|a|)$ and center $a$ in $D$. We can find $a$ sufficiently close to $\partial D$ such that for any choice of $\eta, 0<\eta<1$, the disc $D_{\eta}(a)$ is completely included in the hole in the range of $\phi$. But then, as above, $\tau_{\phi}(z)=0$ on $D_{\eta}(a)$ and so $m\left(G_{c}^{\phi} \cap D_{\eta}(a)\right)=0$ for every positive constant $c$.

Remark 1. If $\phi$ is a univalent function, if $C_{\phi}$ does not have a closed range, and if $\psi$ is such that $\psi(D) \subset \phi(D)$, then $C_{\psi}$ does not have a closed range. This is true because if we let $\omega=\phi^{-1} \circ \psi$, then $\omega$ maps $D$ into itself and $\psi=\phi \circ w$. For a sequence $\left\{f_{n}\right\}$ in $H^{2}$ with $\left\|f_{n}\right\|=1$ and with $\left\|C_{\phi} f_{n}\right\| \rightarrow 0$ we have

$$
\left\|C_{\psi} f_{n}\right\|=\left\|f_{n} \circ \psi\right\|=\left\|f_{n} \circ \phi \circ \omega\right\| \leq\left\|C_{\omega}\right\| \cdot\left\|f_{n} \circ \phi\right\| \rightarrow 0 .
$$

Remark 2. Let $\phi$ be of a bounded multiplicity on some annulus $D \backslash D_{R}=$ $\{z \in D: R<|z|<1\}$, and let $\phi$ be continuous on $\bar{D}$. If there exists $\omega$ 
in $\partial D \cap \phi(\partial D)$ with no finite angular derivative at any of the preimages of $w$, then $C_{\phi}$ does not have a closed range on $H^{2}$.

Proof of Remark 2. Suppose that $C_{\phi}$ has a closed range on $H^{2}$. Then there exists a constant $c>0$ such that $m\left(G_{\phi}^{c}=\{w: \tau(w)>c\} \cap D(\omega, r)\right) \neq 0$ for all $r$ between 0 and 1 . Also the sets $E_{n}=G_{\phi}^{c} \cap D(\omega, 1 / n)$ are nonempty for all $n$. Let $w_{n} \in E_{n}$. Then $w_{n} \rightarrow \omega, w_{n} \in \phi(D)$ and for a sufficiently big $n$, we have $w_{n} \in D \backslash D_{R}$. Let $z_{n}$ be chosen to have the smallest modulus among the elements of $\phi^{-1}\left(w_{n}\right)$, and let us suppose without loss in generality that the sequence $\left\{z_{n}\right\}$ converges to a point $\xi$ on $\partial D$. (We always have that $\left|z_{n}\right| \rightarrow 1$ and so we may work with a subsequence if necessary.) Then $\phi\left(z_{n}\right)=w_{n} \rightarrow \omega$ and because $\phi$ is continuous on $\partial D$, we get $\phi(\xi)=\omega$.

Let $M$ be a bound for the multiplicity of $\phi$ on $D \backslash D_{R}$ and let $z_{n}^{k}, k \leq M$, be all the elements of $\phi^{-1}\left(w_{n}\right)$. Then

$$
c<\tau_{\phi}\left(w_{n}\right)=\frac{\sum_{k} \log \left(1 /\left|z_{n}^{k}\right|\right)}{\log \left(1 /\left|w_{n}\right|\right)} \leq M \frac{\log \left(1 /\left|z_{n}\right|\right)}{\log \left(1 /\left|w_{n}\right|\right)}
$$

for all $n$.

Near $\partial D$ we may replace $\log (1 /|w|)$ by $1-|w|$ and we get

$$
\left(1-\left|z_{n}\right|\right) /\left(1-\left|w_{n}\right|\right)>c / M
$$

for all $n$, i.e., that

$$
\left(1-\left|\phi\left(z_{n}\right)\right|\right) /\left(1-\left|z_{n}\right|\right)<M / c
$$

for all $n$.

But then we have

$$
\liminf \left\{\left(1-\left|\phi\left(z_{n}\right)\right|\right) /\left(1-\left|z_{n}\right|\right): z_{n} \rightarrow \xi\right\} \leq M / c .
$$

On the other hand, $\phi$ does not have a finite angular derivative at $\xi$. By the Julia-Carathéodory Theorem the last statement is equivalent to

$$
\lim \inf \{(1-|\phi(z)|) /(1-|z|): z \rightarrow \xi \text { unrestrictedly in } D\}=\infty .
$$

This contradicts the previous inequality; so $C_{\phi}$ does not have a closed range on $H^{2}$.

Note that we may avoid the requirement of continuity of $\phi$ on $\partial D$ by imposing a new angular derivative criterion on $\phi: \phi$ does not have a finite angular derivative at any point of $\partial D$. But then we are in a much more restrictive situation. By a result of Shapiro [8] bounded multiplicity and the angular derivative criterion on $\phi$ imply that $C_{\phi}$ is compact on $H^{2}$ and so it does not have a closed range.

Example 3. If the range of $\phi$ on $D$ is such that $\phi(D) \subset D \backslash[0,1)$, then $C_{\phi}$ does not have a closed range on $H^{2}$.

Bearing in mind Remark 1, we consider the map $\phi$ which is a univalent map from $D$ onto $D \backslash[0,1]$ satisfying $\phi^{-1}(1)=\{-1,1\}$. The map $\phi$ does not have a finite angular derivative at both 1 and -1 , and so, by Remark $2, C_{\phi}$ does not have a closed range on $H^{2}$.

All the above examples of composition operators $C_{\phi}$ without closed range are such that $\phi(D)$ omits a subset of $D$. What happens if $\phi(D)=D$ ? If $\phi$ 
is the identity map or if $\phi$ is a Möbius transformation then the range of $C_{\phi}$ is closed since $C_{\phi} H^{2}=H^{2}$. Nevertheless, the following example shows that $\phi(D)=D$ does not imply that $C_{\phi}$ has a closed range.

Example 4. Let $\Omega$ be the lens domain bounded by the upper semicircle and by a circular arc (in the lower semidisc) making an angle $\alpha, 0<\alpha<\pi / 2$, with the real axis at the points -1 and 1 . Let $\psi$ be the univalent map from $D$ onto $\Omega$ fixing the points -1 and 1 and take $\phi$ to be $\psi^{2}$. Then $\phi(D)=D$, $\phi$ is of multiplicity at most 2 on $D$, and $\phi$ is continuous on $\bar{D}$. Moreover $\phi^{-1}(1)=\{-1,1\}$ and $\phi$ does not possess a finite angular derivative either at -1 or at 1 . By Remark $2, C_{\phi}$ does not have a closed range on $H^{2}$.

The results on closed-range composition operators are connected with the results on dense range composition operators. The main reason is that every composition operator is one-to-one and the invertible composition operators are induced by disc automorphisms. Akeroyd [1] gives an example of an onto mapping $\phi: D \rightarrow D \backslash \Gamma$ ( $\Gamma$ is a Jordan arc in $D$ with endpoints 0 and 1$)$ for which $C_{\phi}$ has a dense range on $H^{2}$. Hence, this $C_{\phi}$ does not have a closed range.

Mergelyan and Tamadjan have shown [6] that if sufficiently many slits are put in the unit disc, one can obtain a domain $G$ such that if $\phi$ maps $D$ onto $G$ then $C_{\phi}$ has a dense range on $H^{2}$. So $C_{\phi}$ does not have a closed range in this case too. We note though that the mapping $\phi$ from Example 3 above induces $C_{\phi}$ that does not have a dense range on $H^{2}$.

Remark 3. The last remark that we would like to make concerns the general weighted Hardy spaces. Without going into details, let us just point out that disc automorphisms do not induce bounded composition operators on some very small or some very big weighted Hardy spaces. On the spaces where they do induce bounded composition operators, the operators are invertible and so have a closed range.

The next most natural candidate for a closed-range composition operator on every weighted Hardy space is the function $\phi$ defined by $\phi(z)=z^{2}$. But the situation is quite discouraging: on some very small weighted Hardy spaces $\phi$ induces an unbounded composition operator, while, on the other hand, on some very big weighted Hardy spaces it induces a compact composition operator and so its range cannot be closed.

\section{REFERENCES}

1. J. Akeroyd, Density of the polynomials in the Hardy space of certain slit domains, Proc. Amer. Math. Soc. (to appear).

2. J. A. Cima, J. Thompson, and W. R. Wogen, On some properties of composition operators, Indiana Univ. Math. J. 24 (1974), 215-220.

3. C. C. Cowen, Composition operators on Hilbert spaces of analytic functions: A status report, Proc. Sympos. Pure Math., vol. 51, part 1, Amer. Math. Soc., Providence, RI, 1990.

4. D. H. Luecking, Inequalities on Bergman spaces, Illinois J. Math. 25 (1981), 1-11.

5. B. D. MacCluer and J. H. Shapiro, Angular derivatives and compact composition operators on the Hardy and Bergman spaces, Canad. J. Math. 38 (1986), 878-906.

6. S. N. Mergeljan and A. P. Tamadjan, On compactness in a class of non-Jordan regions, Trans. Amer. Math. Soc. 35 (1964), 79-94. 
7. E. A. Nordgren, Composition operators, Canad. J. Math. 20 (1968), 442-449.

8. J. H. Shapiro, The essential norm of a composition operator, Ann. of Math. (2) 125 (1987), 375-404.

Department of Mathematics, University of Manitoba, Winnipeg, Manitoba, R3T 2N2, CANADA

E-mail address: zorbosk@ccu.umanitoba.ca 